\title{
Operational sex ratio does not influence the evolution of male mate choice in the Indian meal moth
}

\author{
Liam R. Dougherty $^{1}$ (1) $\cdot$ Andrea D. Dewhurst ${ }^{1} \cdot$ Zenobia Lewis $^{1}$
}

Received: 4 February 2019 / Accepted: 22 August 2019 / Published online: 29 August 2019

(c) The Author(s) 2019

\begin{abstract}
The strength of mate choice, pre-copulatory sexual selection more generally, is predicted to vary according to a range of social and demographic factors, including the operational sex ratio (OSR). The OSR influences the number of mating opportunities available to each sex, and can, therefore, strongly influence the degree of intrasexual competition and the cost of mate choice. Accordingly, many animals show short-term plasticity in the strength of mate choice in relation to changes in the OSR. However, whether such change persists over evolutionary time has rarely been tested experimentally. In this study, we examined how female mating success in relation to larval food level and body size differs according to the evolutionary history of their mates, in the Indian meal moth Plodia interpunctella. We used males from populations that have been reared at different adult sex ratios (and, therefore, OSRs) for 130+ generations. We found that small females were more likely to mate compared to large females; however, this effect could not be attributed exclusively to male mate choice. Larval food level also did not influence female mating success. Further, males from different sex ratio regimes did not differ in their likelihood of mating with small females, or those from high-food populations. We suggest that male post-copulatory choice is potentially more important than pre-copulatory choice in this species.
\end{abstract}

Keywords Mate choice $\cdot$ Sex ratio $\cdot$ Experimental evolution $\cdot$ Plodia interpunctella $\cdot$ Body size $\cdot$ Sexual selection $\cdot$ Dichotomous choice

\section{Introduction}

Mate choice involves the acceptance of some partners as mates and the rejection of others, and is virtually ubiquitous across the animal kingdom (Andersson 1994; Bonduriansky 2001; Rosenthal 2017). Mate choice is favoured when potential partners vary in quality in some way, so that choosers can gain direct or indirect (genetic) benefits from mating preferentially with high-quality individuals (Andersson 1994; Kokko et al. 2003). However, there are also potential costs associated with mate choice, including time and energy costs associated with searching for and assessing potential partners (Milinski and Bakker 1992; Jennions and Petrie 1997), and the risk of remaining unmated if acceptable partners are not encountered (Barry and Kokko 2010; Greenway et al. 2015). Importantly, the potential costs and benefits of

Liam R. Dougherty

liam.dougherty@liverpool.ac.uk

1 Institute of Integrative Biology/School of Life Sciences, University of Liverpool, Liverpool L69 7RB, UK mate choice are not constant, but can vary depending on the social or ecological environment (Jennions and Petrie 1997). This means that the optimal strength of choice for a given trait (or "choosiness" following the terminology of Reinhold and Schielzeth 2015) may depend strongly on the environment. Because of this, animals frequently exhibit plasticity in choosiness in relation to a range of factors that can influence the costs and benefits of choice (Jennions and Petrie 1997; Dougherty and Shuker 2015; Ah-King and Gowaty 2016). For example, the number of available mating opportunities is an important factor for determining the economics of choice, as it influences the risk of remaining unmated if suitable partners are not encountered (Jennions and Petrie 1997; Barry and Kokko 2010). The number of available mating opportunities is often strongly dependent on the adult sex ratio, or, more correctly, the operational sex ratio (OSR). The OSR represents the ratio of sexually active members of each sex in the population (Kvarnemo and Ahnesjo 1996), and represents the degree of competition for access to mates: intrasexual competition is stronger in sex which is more common. Accordingly, experimental 
manipulation of the OSR in several species has shown that mate choice is stronger in sex which is less common (Berglund 1994; Jirotkul 1999), and in extreme cases, changes in the OSR can lead to a complete reversal in sex which is choosy (Gwynne and Simmons 1990; Forsgren et al. 2004).

As well as examining how behaviours change in response to social and ecological conditions, we can also use an experimental evolution approach to examine how such behaviours change over evolutionary time (Kawecki et al. 2012). Experimental evolution is a powerful technique because it allows us to test whether changes in the environment lead to evolutionary responses, or whether there are other selection pressures which prevent this. Experimental evolution studies have shown that mating preferences can evolve following changes in larval diet (Dodd 1989; Rundle et al. 2005) and artificial selection on ornaments (Brooks and Couldridge 1999). They have also shown that other aspects of mating behaviour, including mating rate, can evolve in response to changes in the population sex ratio (Snook et al. 2005; Harano and Miyatake 2007; Ingleby et al. 2010; Fritzsche et al. 2016). However, we know of only one study that has examined changes in mating preferences in relation to a specific focal trait following experimental manipulation of the population sex ratio. Sales et al. (2018) manipulated adult sex ratio in the flour beetle Tribolium castaneum for 82-106 generations, and then examined the frequency of male-male mating attempts, which may arise due to failures in mate recognition or a reduction in choosiness in relation to partner sex. They show that males from female-biased lines attempted to mate more frequently with other males compared to males from male-biased lines, possibly because there is reduced selection on males in female-biased lines to discriminate between the sexes. More widely, however, it remains unclear whether changes in the social environment can drive changes in the strength of mating preferences over multiple generations, and how rapidly these changes can occur.

In this study, we test whether the strength of male mate choice has evolved in response to experimental manipulation of the adult sex ratio in the Indian meal moth Plodia interpunctella. We used populations of $P$. interpunctella that have been maintained under a male-biased (MB; 3 males: 1 female) or a female-biased (FB; 1 male: 3 females) sex ratio regime for over 130 generations [the generation number differs between populations mainly because development time is slightly faster for male-biased lines (Dougherty, unpublished data)]. These populations differ in the number of available mating opportunities available to each sex, in addition to mating rate: males gain more matings in the female-biased regime than the male-biased regime, and vice versa (Ingleby et al. 2010). Further, these populations have diverged in a number of traits related to mating and reproduction, including male ejaculate investment (Ingleby et al. 2010), female reproductive anatomy (McNamara et al. 2019), and male and female immune activity (McNamara et al. 2013). We compare the mating success of females when mating with males from populations maintained at three different adult sex ratios. We examine male mate choice in relation to two female traits: body condition and body size. To manipulate female body condition, females were reared at two different larval food levels, and males were given a choice between a high-food and a low-food female during mating trials. After the trials, we also measured the wing of length of those same females to test whether female mating success was related to size. Differences in the strength of pre-copulatory sexual selection acting on females between males from different sex ratio regimes would provide evidence that male mate choice has evolved in these populations. Males in this species have been shown to allocate sperm strategically in response to female body size (Gage 1998; but see Ingleby et al. 2010), relatedness (Lewis and Wedell 2009), and age and mating history (Cook and Gage 1995). However, male pre-copulatory choice is yet to be examined in this species.

We have two main questions. First, does female mating success depend on larval food level? Second, does this relationship depend on the evolutionary history, in terms of population sex ratio, of her mating partner? We have three main predictions. First, we predict that males should prefer to mate with females that were reared at a high larval food level, as these females are assumed to be in better condition. Second, males should prefer to mate with larger females, as female size is positively correlated with fecundity in this species (Gage 1998; Lewis et al. 2011). Third, we predict that pre-copulatory sexual selection acting on females should be stronger when males originate from female-biased populations, as males here have more potential mating opportunities and so should be more selective with regard to mate size or condition.

\section{Methods}

We tested for male mating preferences using laboratoryreared males from seven replicate lines of $P$. interpunctella: three lines subject to a male-biased selection regime (MB1, MB2 and MB3), three lines subject to a female-biased selection regime (FB1, FB2 and FB3) and one line maintained at an equal sex ratio. Effective population size was calculated using the average female mating rate presented in Ingleby et al. (2010), and the equation from Caballero (1994). Assuming a conservative estimate of 100 individuals per generation resulted in an approximate effective population size of 67.6 for male-biased lines, 64.2 for equal sex ratio lines, and 59.6 for female-biased lines. All selection lines were initiated in 2004. The equal sex ratio (stock) population originated from individuals collected in Perth, 
Australia, in 2001. Larvae were reared on a standard diet of bran, yeast, organic honey and glycerol, maintained at $28^{\circ} \mathrm{C}$ with a 16:8-h light:dark cycle (Cook and Gage 1995). The selection lines have been maintained in the laboratory for over 10 years (for full methodology, see Ingleby et al. 2010). Briefly, the adult sex ratio is manipulated by introducing adults (between 100 and 120 adults per generation) into mating pots at the correct ratio (stock populations maintained at 1:1 sex ratio). Adults are then allowed to mate for $72 \mathrm{~h}$, and eggs are collected into a pot below separated by gauze. After 72 h, 400 eggs are randomly sampled to establish the next generation. Therefore, larval sex ratio and density are the same for all populations. Due to divergence in the average development time, the exact generation number varies across populations; however, males from each replicate line were taken from a single-generation only. We tested males from generations 133 (FB3), 134 (FB1, FB2, MB3), 135 (MB2) and 143 (MB1).

To control for female effects, all males were tested using females from the stock population. We manipulated larval food level for stock females by creating two food treatments, each having the same larval density (60 eggs per pot), but with either high or low food (high: $20 \mathrm{~g}$ of food per pot, $333 \mathrm{mg}$ per larvae; low: $4 \mathrm{~g}$ of food per pot, $66 \mathrm{mg}$ per larvae). All experimental males were reared at the same density as the high-food treatment. Larvae were sexed and separated into individual tubes containing a small amount of food (larvae are more likely to pupate when food is present) after 14 days to ensure virginity. These tubes were checked daily for the presence of adults to accurately determine age.

To assess male mating preference, we used a dichotomous choice design, with two females introduced into a universal tube with a male, with one female from each larval food level. To distinguish between the females, a small portion of the wing was cut from one of the females the day before a trial under $\mathrm{CO}_{2}$ anaesthesia. We randomly allocated females so that half of the cut females were from the low-food level and half from the high-food level. The females that were not cut were also anaesthetised as a sham control. During trials, the observer was blind to the female food level. In each tube, the females were matched for age, and only receptive females were used in trials (observed in the 'calling' position either on the previous day or on the morning of the trial). All individuals were virgins aged between 1 and 4 days post-adult eclosion. All trials were performed in the first $3 \mathrm{~h}$ of the dark phase (10 a.m.-1 p.m.), under ambient light and at room temperature. We recorded the mating latency and which female mated. Trials were ended after $30 \mathrm{~min}$ if the male failed to mate with either female $(N=25)$. Mating pairs were allowed to finish copulation naturally (this takes between 60 and $120 \mathrm{~min}$ ), and then all mated and unmated individuals were euthanised. We performed 270 mating trials in total.
For mating trials in which one female mated ( 245 out of 270 trials), we measured the size of one of the forewings for all individuals, as a proxy for body size (Lewis et al. 2011). Wings were removed and dipped briefly in $\mathrm{HCl}$ and then bleach to remove scales. Wings were then placed on a microscope slide and photographed using a mobile phone attached to a dissecting microscope. In the majority of cases, we removed the left forewing (428 out of $486,88.1 \%$ ); however, there was no significant difference in length between the left and right forewings ( $t$ test, $t=-1.009, N=486, P=0.316)$. Wing lengths were measured using the software package ImageJ (Schneider et al. 2012). We measured a sample of wings twice, and found that measurements were highly repeatable $(N=36$, $\mathrm{R}=0.983)$. We obtained morphological data for 242 mated males (25-32 males from each replicate selection line and 68 from the stock population) and 486 females ( 243 trials).

We tested for the presence of male mate choice in two ways. First, we examined whether high- or lowfood females were more likely to mate, using only trials in which one female mated. We ran a generalised linear mixed model with mated female coded as a binary response variable (high or low food). Sex ratio regime and male size were included as fixed factors in this model, and male replicate line was included as a random factor, nested within sex ratio regime. Second, we analysed female mating success with female individual (not trial; because we did not control for female size during trials) as the unit of analysis, using a generalised linear mixed model with mating success (mated or unmated) as a binary response variable, and tube (243 trials) and male replicate line (nested within sex ratio regime) as random effects. We included sex ratio regime, female food level, female cutting treatment, female size, and the interaction between female size and sex ratio regime, as fixed factors. We also present the results of a simplified model after excluding two fixed factors (sex ratio regime and the interaction effect) which had low explanatory power. The significance of all fixed effects was tested using type III analysis of variance. To visualise the (potentially non-linear) shape of sexual selection on female size, we produced fitness surfaces using nonparametric cubic splines (Schluter 1988). Curves were calculated using general additive models using the package MGCV v1.8 (Wood 2011), including female wing length as the single predictor variable and the smoothing parameter obtained by minimising the generalised crossvalidation (GCV) score of the GAM.

We also tested for differences in mating latency (irrespective of which female was chosen), using a linear mixed model, with male replicate line as a random factor nested within sex ratio regime, and sex ratio regime, male size, and male and female age (in days) as fixed factors. Latency data were log-transformed prior to analysis. All statistical 
analyses were performed in $\mathrm{R}$ version 3.5 (R Development Core Team 2018).

\section{Results}

245 out of 270 mating trials resulted in 1 of the females mating. Across all trials, high-food females were no more likely to mate than low-food females (males mated with high-food females in 128 out of 245 trials; Chi-square test: $\chi^{2}=0.49$, $d f=1, P=0.48$; Fig. 1). Further, the proportion of males mating with high-food females did not differ across the three sex ratio regimes (GLMM: $\chi^{2}=0.54, d f=2, P=0.76$ ). The likelihood of a male mating with a high-food female was not influenced by male size $\left(\chi^{2}=2.5, d f=1, P=0.11\right)$. Posttrial wing measurements revealed that there was no difference in size between high-food and low-food females ( $t$ test, $t=-1.047, N=243$ high food, 243 low food, $P=0.29$ ). This means that female body size is not confounded by larval food level, and so may separately influence female mating success.

We next examined predictors of female mating success, this time using all females $(N=486$ females from 243 trials). Smaller females were more likely to mate (Fig. 2), and this relationship was significant for the minimal model, but not for the full model (Table 1). However, this relationship was not influenced by the sex ratio regime experienced by males (Table 1: interaction between female size and male sex ratio). Further, female mating success was also influenced by wing cutting, with cut females more likely to mate (Table 1 ; 243 mated females, 145 cut and 98 uncut). This effect is not due to a size difference between cut and uncut females ( $t$ test, $t=1.3, N=486, P=0.19$ ).
We also tested for differences in male mating latency, irrespective of which female was chosen in a trial. Logtransformed male mating latency $(N=242$ trials $)$ was not significantly influenced by sex ratio regime $\left(\chi^{2}=1.18, d f=2\right.$, $P=0.55$; Fig. 3$)$, male size $\left(\chi^{2}=1.8, d f=1, P=0.18\right)$, male age $\left(\chi^{2}=2.4, d f=1, P=0.12\right)$ or female age $\left(\chi^{2}=2.61\right.$, $d f=1, P=0.11)$.

\section{Discussion}

This study tested two main questions in relation to male mate choice in the Indian meal moth Plodia interpunctella. First, is there pre-copulatory sexual selection in relation to female body size or condition in this species? Second, has the strength of male mate choice for these traits evolved in response to contrasting adult sex ratios over $140+$ generations? Female larval food level (a proxy for adult condition) did not influence female mating success, irrespective of the evolutionary history of her mating partner. We found that smaller females were more likely to mate across all replicate lines; however, this relationship was not influenced by the sex ratio regime experienced by males, despite large differences in the number of available mating opportunities across regimes. This result, coupled with the fact that our experimental design does not allow us to explicitly attribute choice to either sex, suggests that male mate choice for female condition or body size is weak in these populations. We also found no difference in overall male mating latency in relation to male sex ratio regime.

We found evidence for pre-copulatory sexual selection across our experimental populations: smaller females were more likely to mate following dichotomous choice tests.
Fig. 1 Female mating success (proportion of trials mated) in relation to larval food level, across the seven male replicate lines. Filled bars are high-food females, open bars are low-food females. $F B$ female-biased sex ratio, stock equal sex ratio, $M B$ male-biased sex ratio. Sample sizes $=29,26$ and 28 trials for FB1, FB2, and FB3 respectively; 68 trials for stock; and 32, 30 and 32 trials for MB1, MB2 and MB3, respectively. All females originated from an equal sex ratio population

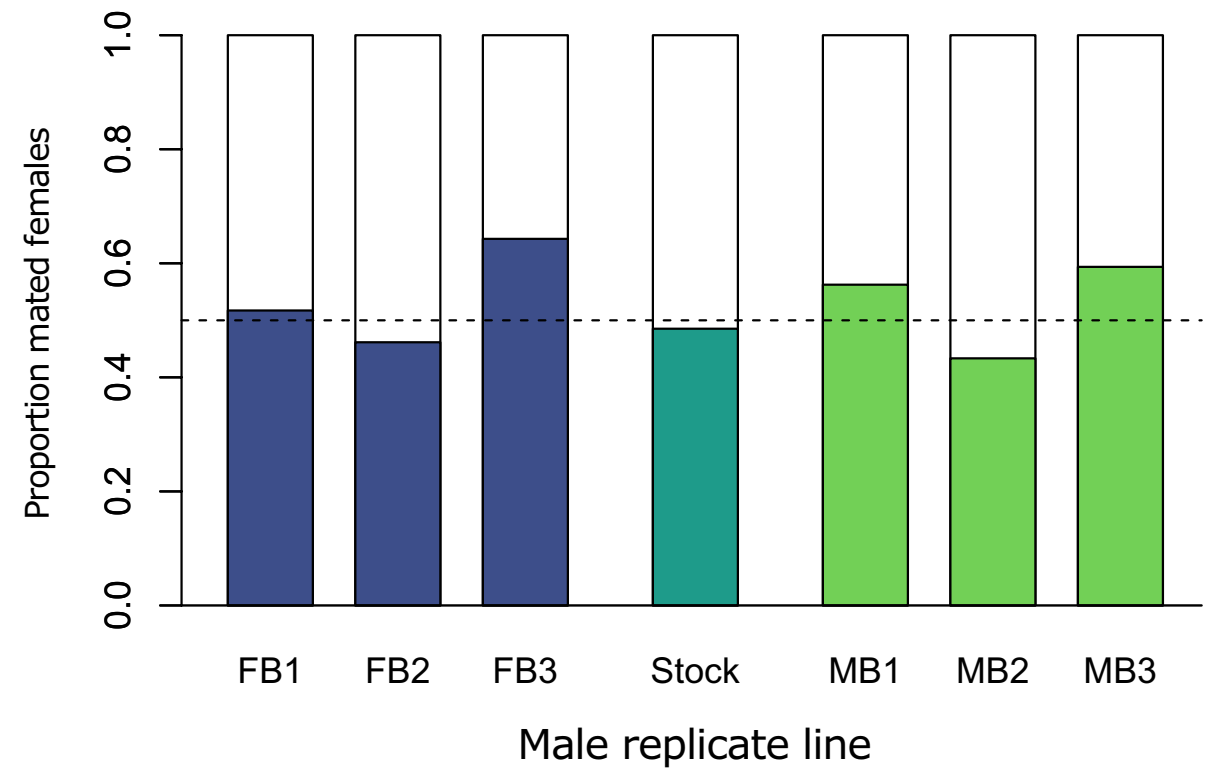


Fig. 2 Relationship between female wing length and mating success $(0=$ unmated, $1=$ mated) for stock females mating with males from all replicate lines combined $(N=243$ mated and 243 unmated females). Fitted curve is a cubic spline (dotted lines shows \pm 1 standard error) fitted using a general additive model, see text for details. Scale for histogram not shown

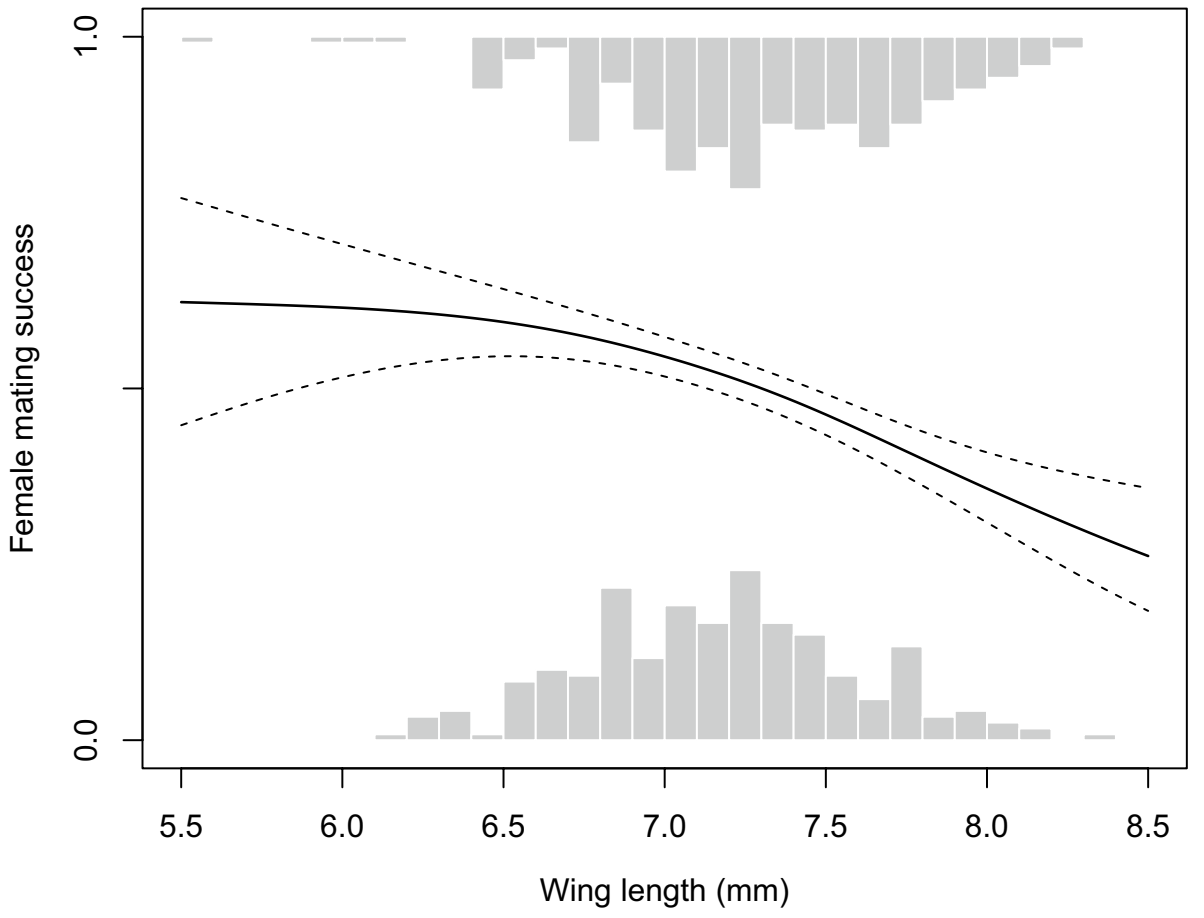

Table 1 Determinants of female mating success across all trials $(N=243$ trials and 486 females in both cases)

\begin{tabular}{lccc}
\hline Factor & $\chi^{2}$ & $d f$ & $P$ \\
\hline Full model & & & \\
$\quad$ Intercept) & 0.49 & 1 & 0.48 \\
Body size & 0.3 & 1 & 0.59 \\
Food level & 0.54 & 1 & 0.46 \\
Male sex ratio regime & 2.1 & 2 & 0.35 \\
Wing cutting & 20.08 & 1 & $<0.001$ \\
Body size $\times$ male sex ratio regime & 2.13 & 2 & 0.35 \\
Minimal model & & & \\
(Intercept) & 12.55 & 1 & $<0.001$ \\
Body size & 10.96 & 1 & $<0.001$ \\
Wing cutting & 19.85 & 1 & $<0.001$ \\
\hline
\end{tabular}

The full model (left) includes all relevant predictors and interactions, including female food level (243 high-food females and 243 lowfood females), male sex ratio regime ( 83 males from female-biased lines, 66 males from stock, and 94 males from male-biased lines), and female wing cutting treatment (243 cut, 243 sham). The minimal model (right) shows the remaining predictors are removing non-significant interactions and the main effect of male sex ratio regime. The significance of fixed effects was determined using Type III analysis of variance

This is in the opposite direction to our prediction that larger females should be preferred by males, given that they have a higher reproductive potential (Gage 1998; Lewis et al. 2011), and that males allocate more sperm to larger females during mating (Gage 1998). Why would smaller females be more likely to mate in this species? One problem with interpreting our results is that our experimental design does not test male mate choice directly; because males and females were able to fully interact before mating, we cannot rule out the possibility that females also contribute to mating decisions (Dougherty and Shuker 2014). Female mate choice has not been studied in this species, but this pattern could arise if smaller females were more likely to accept matings than large females, perhaps because the latter have higher fecundity and so may be choosier. Courtship involves shortrange male pheromone (Grant 1974) and ultrasound production (Trematerra and Pavan 1995), but the actual behavioural interactions between males and females are very brief. However, successful coupling does require female cooperation, and females are able to reject matings (Grant 1974; Lewis et al. 2013). Alternatively, the observed pattern could also be due to smaller females being less able to resist forced matings, as seen in other species (Arnqvist and Rowe 2005). There is little evidence for active mating struggles in this species. However, the last stage of courtship involves the male attempting to grab the end of the female abdomen with his genital claspers (valvae) while both are in a face-to-face position (Grant and Brady 1975), a manoeuvre which would likely be easier if the female was small and so closer to his size. The importance of female resistance is also supported by the fact that females were more likely to mate when they had a portion of the wing removed before the trial. It is not clear why this would make females more attractive to males, but it could possibly make females less able to resist male mating attempts by hindering their ability to escape. 
Fig. 3 Mating latency (natural $\log$, in seconds) of mated males in relation to male replicate line. Sample sizes $=29,26$ and 28 males for FB1, FB2, and FB3, respectively; 68 males for stock; and 32, 30 and 32 males for MB1, MB2 and MB3, respectively. All females originated from an equal sex ratio population

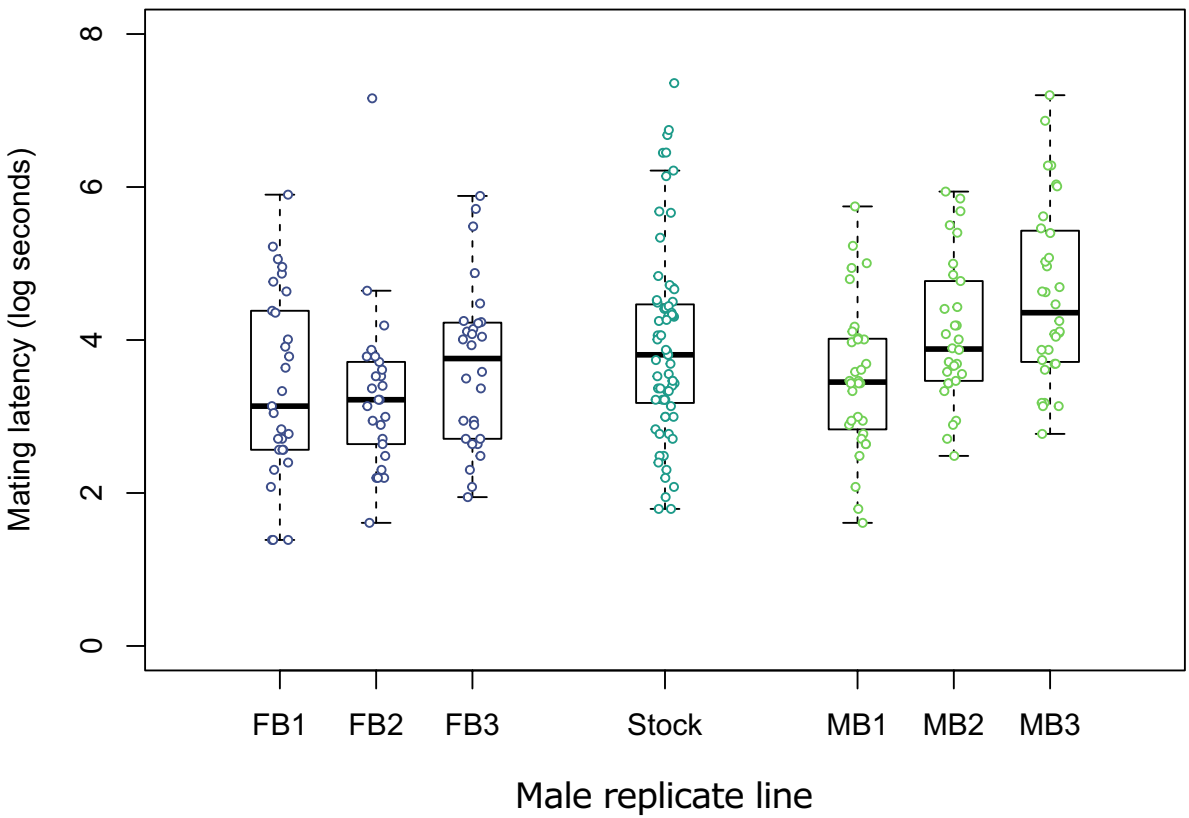

Overall, the results suggest that male mate choice with respect to either female body size or condition is weak. This is surprising given the biology of this species. P. interpunctella do not feed as adults; all spermatogenesis occurs during larval development (Cook and Gage 1995), so that males have a limited sperm supply that cannot be replenished during adulthood. This life-history strategy as well as the potential reproductive benefits associated with mating with larger females (Gage 1998; Lewis et al. 2011) both support the idea that mate choice would benefit males in this species. However, female mating success was only weakly related to condition or body size, suggesting that male mate choice in relation to these traits is very weak. This is surprising given that males have been shown to exhibit post-copulatory choice in relation to female body size (Gage 1998), suggesting that males may be able to assess this in females. One reason for this could be that male mating success more strongly depends on a high mating rate or a high level of polygyny. Males have been recorded as mating up to eight times in 8 days in staged mating trials (Ryne et al. 2001). However, in our experimental populations, the male mating rate is more modest, averaging only three matings in the female-biased lines (Ingleby et al. 2010). Alternatively, in wild populations, it may be that long-range male attraction to pheromone-emitting females is more important than shortrange mating interactions (Zhu et al. 1999). However, such long-range signalling is probably not important in our lab populations, given the high densities and small pots used for maintenance $\left(1045 \mathrm{~cm}^{3}\right)$.

Another possibility is that males may have historically been selected to accept essentially all matings, and then strategically allocate sperm during mating. This is supported by the fact that male post-copulatory choice appears much stronger than pre-copulatory choice, with males being much more prudent when it comes to sperm allocation in relation to female size, age, mating history and relatedness (Cook and Gage 1995; Gage 1998; Lewis and Wedell 2009). However, we must note that male mate choice in relation to the latter three traits has not been tested in this species. This difference between pre- and post-copulatory choice could arise, for example, if males are unable to assess female state before mating.

We did not detect any changes in male mating latency or choosiness due to the differing sex ratio regimes. This is surprising given that males gain four times as many matings on average in female-biased lines compared to male-biased lines (Ingleby et al. 2010), and theory suggests males should become choosier as their expected number of mating opportunities increases (Jennions and Petrie 1997). In light of the overall results, this could be explained by the fact that male mate choice in the ancestral population is weak, so that there is no preference that selection is able to act on. Further, any selection favouring pre-copulatory choice arising from the sex ratio regime would have to be strong enough to overcome selection favouring post-copulatory selection, such as the high level of sperm competition faced by males. If selection favouring post-copulatory choice is strong, it could be that a more biased sex ratio regime (e.g. 10:1) would be needed to favour the evolution of pre-copulatory choice.

This study provides a rare examination of whether mate choice evolves in response to consistent changes in the costs and benefits of choice over multiple generations. We detected no difference in male mate choice or mating latency between selection lines maintained at contrasting operational 
sex ratios. This is surprising given the importance of the OSR for driving short-term changes in choosiness (Jennions and Petrie 1997; Ah-King and Gowaty 2016), as well as being a key determinant of the strength of sexual selection acting on each sex (Janicke and Morrow 2018). Nevertheless, we suggest that the evolvability of mate choice should be investigated further, both in relation to the operational sex ratio and to other factors that influence the economics of mate choice, such as predation risk or population density (Jennions and Petrie 1997; Ah-King and Gowaty 2016). Such work is needed to bridge the gap between the many studies showing short-term plasticity in choosiness, and long-term population-level changes. Doing so will help us to better elucidate the conditions favouring the origin and maintenance of mating preferences over evolutionary time.

Acknowledgements This work was supported by the Association for the Study of Animal Behaviour (Research grant to LRD).

Open Access This article is distributed under the terms of the Creative Commons Attribution 4.0 International License (http://creativeco mmons.org/licenses/by/4.0/), which permits unrestricted use, distribution, and reproduction in any medium, provided you give appropriate credit to the original author(s) and the source, provide a link to the Creative Commons license, and indicate if changes were made.

\section{References}

Ah-King M, Gowaty PA (2016) A conceptual review of mate choice: stochastic demography, within-sex phenotypic plasticity, and individual flexibility. Ecol Evol 6:4607-4642

Andersson MB (1994) Sexual selection. Princeton University Press, Princeton

Arnqvist G, Rowe L (2005) Sexual conflict. Princeton University Press, Princeton

Barry KL, Kokko H (2010) Male mate choice: why sequential choice can make its evolution difficult. Anim Behav 80:163-169

Berglund A (1994) The operational sex ratio influences choosiness in a pipefish. Behav Ecol 5:254-258

Bonduriansky R (2001) The evolution of male mate choice in insects: a synthesis of ideas and evidence. Biol Rev 76:305-339

Brooks R, Couldridge V (1999) Multiple sexual ornaments coevolve with multiple mating preferences. Am Nat 154:37-45

Caballero A (1994) Developments in the prediction of effective population size. Heredity 73:657-679

Cook PA, Gage MJG (1995) Effects of risks of sperm competition on the numbers of eupyrene and apyrene sperm ejaculated by the moth Plodia interpunctella (Lepidoptera: Pyralidae). Behav Ecol Sociobiol 36:261-268

R Development Core Team (2018) Book R: a language and environment for statistical computing. R Foundation for Statistical Computing, Vienna

Dodd DM (1989) Reproductive isolation as a consequence of adaptive divergence in Drosophila pseudoobscura. Evolution 43:1308-1311

Dougherty LR, Shuker DM (2014) Pre-copulatory sexual selection in the seed bug Lygaeus equestris: a comparison of choice and nochoice paradigms. Anim Behav 89:207-214
Dougherty LR, Shuker DM (2015) The effect of experimental design on the measurement of mate choice: a meta-analysis. Behav Ecol 26:311-319

Forsgren E, Amundsen T, Borg ÅA, Bjelvenmark J (2004) Unusually dynamic sex roles in a fish. Nature 429:551-554

Fritzsche K, Booksmythe I, Arnqvist G (2016) Sex ratio bias leads to the evolution of sex role reversal in honey locust beetles. Curr Biol 26:2522-2526

Gage MJG (1998) Influences of sex, size, and symmetry on ejaculate expenditure in a moth. Behav Ecol 9:592-597

Grant G (1974) Male sex pheromone from the wing glands of the Indian meal moth, Plodia interpunctella (Hbn.)(Lepidoptera: Phycitidae). Experientia 30:917-918

Grant G, Brady U (1975) Courtship behavior of phycitid moths. I. Comparison of Plodia interpunctella and Cadra cautella and role of male scent glands. Can J Zool 53:813-826

Greenway EVG, Dougherty LR, Shuker DM (2015) Mating failure. Curr Biol 25:R534-R536

Gwynne DT, Simmons LW (1990) Experimental reversal of courtship roles in an insect. Nature 346:172-174

Harano T, Miyatake T (2007) No genetic correlation between the sexes in mating frequency in the bean beetle, Callosobruchus chinensis. Heredity 99:295-300

Ingleby FC, Lewis Z, Wedell N (2010) Level of sperm competition promotes evolution of male ejaculate allocation patterns in a moth. Anim Behav 80:37-43

Janicke T, Morrow EH (2018) Operational sex ratio predicts the opportunity and direction of sexual selection across animals. Ecol Lett 21:384-391

Jennions MD, Petrie M (1997) Variation in mate choice and mating preferences: a review of causes and consequences. Biol Rev $72: 283-327$

Jirotkul M (1999) Operational sex ratio influences female preference and male-male competition in guppies. Anim Behav 58:287-294

Kawecki TJ, Lenski RE, Ebert D, Hollis B, Olivieri I, Whitlock MC (2012) Experimental evolution. Trends Ecol Evol 27:547-560

Kokko H, Brooks R, Jennions MD, Morley J (2003) The evolution of mate choice and mating biases. Proc R Soc B 270:653-664

Kvarnemo C, Ahnesjo I (1996) The dynamics of operational sex ratios and competition for mates. Trends Ecol Evol 11:404-408

Lewis Z, Wedell N (2009) Male moths reduce sperm investment in relatives. Anim Behav 77:1547-1550

Lewis Z, Wedell N, Hunt J (2011) Evidence for strong intralocus sexual conflict in the Indian meal moth, Plodia interpunctella. Evolution 65:2085-2097

Lewis Z, Lizé A, Wedell N (2013) The interplay between different stages of reproduction in males of the moth Plodia interpunctella. Anim Behav 86:917-922

McNamara KB, Wedell N, Simmons LW (2013) Experimental evolution reveals trade-offs between mating and immunity. Biol Lett 9:20130262

McNamara KB, Dougherty LR, Wedell N, Simmons LW (2019) Experimental evolution reveals divergence in female genital teeth morphology in response to sexual conflict intensity in a moth. J Evol Biol 32:519-524

Milinski M, Bakker TC (1992) Costs influence sequential mate choice in sticklebacks, Gasterosteus aculeatus. Proc R Soc B 250:229-233

Reinhold K, Schielzeth H (2015) Choosiness, a neglected aspect of preference functions: a review of methods, challenges and statistical approaches. J Comp Physiol A 201:171-182

Rosenthal GG (2017) Mate choice. Princeton University Press, Princeton

Rundle HD, Chenoweth SF, Doughty P, Blows MW (2005) Divergent selection and the evolution of signal traits and mating preferences. PloS Biol 3:1988-1995 
Ryne C, Zhu J-W, Van Dongen S, Löfstedt C (2001) Spermatophore size and multiple mating: effects on reproductive success and post-mating behaviour in the Indian meal moth. Behaviour 138:947-963

Sales K, Trent T, Gardner J, Lumley AJ, Vasudeva R, Michalczyk Ł, Martin OY, Gage MJ (2018) Experimental evolution with an insect model reveals that male homosexual behaviour occurs due to inaccurate mate choice. Anim Behav 139:51-59

Schluter D (1988) Estimating the form of natural selection on a quantitative trait. Evolution 42:849-861

Schneider CA, Rasband WS, Eliceiri KW (2012) NIH Image to ImageJ: 25 years of image analysis. Nat Methods 9:671

Snook RR, Robertson A, Crudgington HS, Ritchie MG (2005) Experimental manipulation of sexual selection and the evolution of courtship song in Drosophila pseudoobscura. Behav Genet 35:245-255

Trematerra P, Pavan G (1995) Ultrasound production in the courtship behaviour of Ephestia cautella (Walk.), E. kuehniella Z. and
Plodia interpunctella (Hb.)(Lepidoptera: Pyralidae). J Stored Prod Res 31:43-48

Wood SN (2011) Fast stable restricted maximum likelihood and marginal likelihood estimation of semiparametric generalized linear models. J R Stat Soc B 73:3-36

Zhu J, Ryne C, Unelius CR, Valeur PG, Löfstedt C (1999) Reidentification of the female sex pheromone of the Indian meal moth, Plodia interpunctella: evidence for a four-component pheromone blend. Entomol Exp Appl 92:137-146

Publisher's Note Springer Nature remains neutral with regard to jurisdictional claims in published maps and institutional affiliations. 